\title{
Beam characterization of a microfading tester: evaluation of several methods
}

\author{
Paweł Świt ${ }^{1 *}$ (D, Marco Gargano² and Julio M. del Hoyo-Meléndez ${ }^{3}$
}

\begin{abstract}
Microfading testing allows to evaluate the sensitivity to light of a specific artwork. Characterization of the illumination spot is important to determine its shape, dimensions, light distribution, and intensity in order to limit and account for possible damage. In this research the advantages and disadvantages of several methods used to determine the beam shape and intensity profiles are described with the aim of providing various options to microfading researchers interested in characterizing their irradiation spots. Conventional and imaging methods were employed and are compared in terms of their accuracy, cost, reliability, and technical features. Conventional methods consisted of an aperture technique using aluminium foil and four different materials namely stainless steel, silicon, muscovite, and Teflon used as sharp edges. The imaging methods consisted of digital photography of illumination spot, direct beam measurement using a CMOS camera, and direct beam measurement using a laser beam profiler. The results show that both conventional and imaging methods provide beam width measurements, which are in satisfactory agreement within experimental error. The two best methods were direct measurement of the beam using a CMOS camera and sharp-edge procedure. MFT illumination beam with a CMOS camera followed by a determination of the beam diameter using a direct method, more specifically one involving a sharp-edge technique.
\end{abstract}

Keywords: Beam profile, Illumination intensity, Sharp-edge method, Microfading, Spot shape

\section{Introduction}

Microfading testing (MFT) has become widely accepted by the conservation science community as an adequate tool for establishing and recommending appropriate gallery lighting conditions that minimize damage to collections. These devices offer the opportunity of measuring the photostability of objects due to their optical setup, which allows to conduct and quantify accelerated photoaging over a spot of approximately $0.5 \mathrm{~mm}$. Also, by using a high sensitivity photodetector it is possible to measure spectrocolorimetric change before it is perceived by the human eye. Although a considerable amount of testing is currently performed with these instruments, there are still safety concerns in terms of possible damage to the

\footnotetext{
*Correspondence: pawel.swit@us.edu.pl

${ }^{1}$ Institute of Chemistry, Faculty of Science and Technology, University of Silesia, 40006 Katowice, Poland

Full list of author information is available at the end of the article
}

objects due to the use of a high intensity spot during testing. Nevertheless microfadeometry is widely considered a non-destructive technique providing the evaluation of the light sensitivity of objects over the last two decades [1-8]. This is due to the small spot size employed, which permits the evaluation of the durability of materials, while causing no harm to the object. The process is less time consuming than traditional accelerated light aging tests and the results can be followed in real time. The sensitivity of objects to visible light can be determined by short increments in exposure time and simultaneous direct lightfastness measurements. This equipment offers the opportunity of direct testing the sensitivity to light of objects due to the microscopic size of the area under investigation. Prior to this equipment's development it took weeks to acquire lightfastness data and it was not possible to obtain information directly from the collections of unique objects.

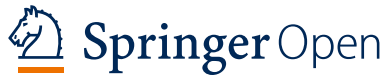

(c) The Author(s) 2021. This article is licensed under a Creative Commons Attribution 4.0 International License, which permits use, sharing adaptation, distribution and reproduction in any medium or format, as long as you give appropriate credit to the original author(s) and the source, provide a link to the Creative Commons licence, and indicate if changes were made. The images or other third party material in this article are included in the article's Creative Commons licence, unless indicated otherwise in a credit line to the material. If material is not included in the article's Creative Commons licence and your intended use is not permitted by statutory regulation or exceeds the permitted use, you will need to obtain permission directly from the copyright holder. To view a copy of this licence, visit http://creativeco mmons.org/licenses/by/4.0/. The Creative Commons Public Domain Dedication waiver (http://creativecommons.org/publicdomain/ zero/1.0/) applies to the data made available in this article, unless otherwise stated in a credit line to the data. 
MFT is a well-established technique and many institutions are aware of the multiple advantages of microfading such as being a unique tool for exhibition planning $[9,10]$. Besides this, it is important to note that there are many institutions, which are not equipped with MFT instruments and have to just rely on illuminance measurements. Additionally, some conservators still consider MFT harmful due to possible photo-oxidation reactions, heating of the sample, and possible interactions of individual chemical components present in the sample area. Other researchers have raised concerns about the differences between the spectral power distribution and intensity of gallery lighting used to illuminate the objects and those used for MFT tests. The authors believe that all these considerations could limit the further development and spread of the MFT technique.

Fortunately, in most cases, thanks also to the relatively short testing time the techniques allows conservators and conservation scientists to make surveys of collections prior to their display in order to determine if there are critical works having unusually high sensitivity to light exposure $[2,4,11]$. The instrument also offers the possibility of performing light-fastness tests on a single material (e.g. powder pigment) or mock-ups prepared in the laboratory consisting of combinations of two or more materials (e.g. pigment/binder system) [12, 13]. Recently there has been considerable interest in the use of MFT and the number of cultural institutions currently using or considering construction or purchase of a MFT device increases steadily [14]. Although some people have expressed concern regarding the safety of objects during and after testing, the technique continues to be widely employed in institutions around the world. In contrast to research-based applications where it is important to accurately determine the power density of the beam, the safety of the object under investigation is of less concern in MFT as the degree of change is monitored in real time and the test can be stopped once a threshold level is reached.

Several authors have pointed out the importance of working with a safe and stable irradiation spot when performing spectroscopic measurements on objects. Due to concerns associated with two-photon effect at high irradiance, local heating leading to local relative humidity change, which could cause significant movement in the $\mathrm{Z}$ direction of the area being microfaded, post-exposure recovery of colour or further colour change after measuring, previous publications have discussed the importance of conducting safe measurements on objects when using techniques such as Raman spectroscopy [15], X-ray fluorescence spectrometry (XRF) [16], optical coherence tomography (OCT) [17], and laser induced breakdown spectroscopy (LIBS) [18], among others. The potential temperature increase at the surface of an object caused by exposure to light from a microfading device has been described by Ford [3], Lerwill [19] and Whitmore [1]. These three studies revealed that the temperature of the investigated surface may experience an increase up to a maximum of $5{ }^{\circ} \mathrm{C}$. Ford has noted that these changes preclude thermal damage to objects or having an influence on fading rates and mechanisms [3]. The concern about initiating two-photon processes has also been expressed in relation to flash photography [20]. However, the author concluded that modern flash units are not powerful enough to initiate two-photon degradation processes, as had been suggested in the past. The illuminance delivered to a white standard can be used to contextualize the discussion on potential photodegradation. For example, white reflectance standards, typically used in calibration of MFT instruments, have a surface with high flat spectral response. It is known that factors such as movement of the light source, fiber optics, or measuring probes may increase the uncertainty of measurements, especially when working at high intensity levels. However, spectral measurements performed on a similar instrument using a barium sulphate white tile did not alter for about $40 \mu \mathrm{m}$ through focus [21]. The authors also indicated that spectral measurement is more sensitive than the variation in size of the illuminated spot with probe position since the alignment of illumination and measurement probes is the key to correct interpretation of the results.

A well characterized spot is essential in microfading research since the technique makes use of a high intensity spot that acts on the object's surface to determine its stability to light. The shape, dimensions, light distribution and intensity of the spot are important parameters to assess and verify non-invasiveness. Therefore, characterizing the irradiation spot is essential to ensure that the tests are performed in a non-invasive and nondestructive way. Safety of the irradiated surface is also a priority for other research applications. For example, a well-defined focused laser spot is especially important for materials processing and medical applications [22, 23]. There are several methods currently used for the determination of the beam profile and diameter. One of the most popular is the knife-edge method, where the power of the laser beam is measured using a photo diode while moving a sharp edge through the laser spot using small distance increments [24]. Although this is a relatively slow technique with a resolution of about $1 \mu \mathrm{m}$, it is still widely employed in many research fields. Another simple and relatively inexpensive method is exposure of thermal or photopaper to the investigated beam in order to produce a visible spot, which can later be accurately measured. A disadvantage of using thermal or photopaper is its low dynamic range and the dependance of 
the measured beam profile on the exposure time [25]. Another commonly used conventional way of determining the beam diameter of a point device is the aperture method. The beam diameter may be determined for a Gaussian beam by positioning an aperture in the center of the beam and measuring the fraction of emitted power passing through the aperture [26].

Other methods involve the use of digital cameras to acquire photographic images of the output beams. Golnabi and Haghighatzadeh have obtained reflection and image transmittance data of an optical beam shaping system based on a plastic fiber-bundle prism-coupled waveguide by using a light emitting diode (LED) source for the illumination and performing image analysis with a charge coupled device (CCD) digital camera [27]. Moreover, complementary metal oxide semiconductor (CMOS) cameras have also been employed to perform laser beam profile measurements [28]. More recent approaches have involved the use of smartphones [29] and webcams [30] as low-cost beam profilers. Characterization of the illumination spot is also important in microfading research since the experiments are conducted on objects sometimes containing extremely sensitive materials. A test conducted by an unexperienced operator on a very sensitive material may result in a visible small spot (estimated range $0.3-0.5 \mathrm{~mm}$ ) on the object as a result of a prolonged exposure to microfading illumination. Examples showing discoloration of colored samples exposed to excessive exposures can be found in $[1,3,31]$. It is important to note that these were extreme tests that were conducted with the aim of producing a clearly detectable color change for visualization purposes. In contrast, microfading tests require much smaller exposures, which can be real-time controlled to prevent such discernable alterations. In addition to experience, a careful selection of the testing areas is recommended along with monitoring and documentation of the tested spot using imaging methods. Liang et al. have measured the profile of the incident spot of a retroreflective microfading spectrometer using a CCD camera finding that the minor axis of the spot was approximately $0.46 \mathrm{~mm}$ full-width at half maximum (FWHM) [12]. Whitmore employed a similar approach to the thermal or photopaper method mentioned above to determine a $0.4 \mathrm{~mm}$ diameter for a test area using his original instrument [1]. Lerwill determined a $0.25 \mathrm{~mm}$ diameter of the incident light, of a custom built microfadometer, acting upon the investigated area through a series of observations using a CCD camera [19]. Pesme et al. developed three portable MFT instruments and compared their performance to that of Whitmore's original design [32]. The study revealed that all instruments operated within safe exposure limits, even when working with a contact technique. The authors report estimated illumination beam diameters, which remained within the $0.5-0.6 \mathrm{~mm}$ range. It was noted that the intensity delivered by each of these three instruments on the tested surface was related to the diameter of the target spot, which was considered difficult to measure accurately. The objective of the present study was to obtain qualitative and quantitative information about the output beam shape of a custom-built MFT instrument and discuss the performance and efficiency of several measuring methods with the aim of providing a range of alternatives to MFT users interested in characterizing their illumination spots.

\section{Experimental}

\section{Microfading tester (MFT)}

The MFT evaluated in this study is a custom-built instrument developed by researchers at the National Museum in Krakow, the Faculty of Physics and the Faculty of Chemistry of the Jagiellonian University [31] and it is based on the original prototype designed by Whitmore et al. [1]. This instrument consists of a high-power light source, a $0^{\circ} / 45^{\circ}$ geometry optical setup, and a Vis reflectance spectrometer that is used to measure the materials' responsiveness upon irradiation. The high-power light source employed is a HPLS 30-04 solid state plasma light source (LIFI) from Thorlabs (New Jersey, US), with emission in the $350-750 \mathrm{~nm}$ range. The spectral power distribution of the light source is shown in Fig. 1. The intensity of the peak at $545 \mathrm{~nm}$ was monitored during the direct measurements performed to characterize the illumination spot.

An OSL 1-EC high intensity fiber light source (Thorlabs, US) with a $380-800 \mathrm{~nm}$ emission range was used in some of the experiments to determine the shape and size of the collection spot. Both sources were filtered to remove wavelengths shorter than $400 \mathrm{~nm}$ and higher than $750 \mathrm{~nm}$. The system uses fiber optics to provide

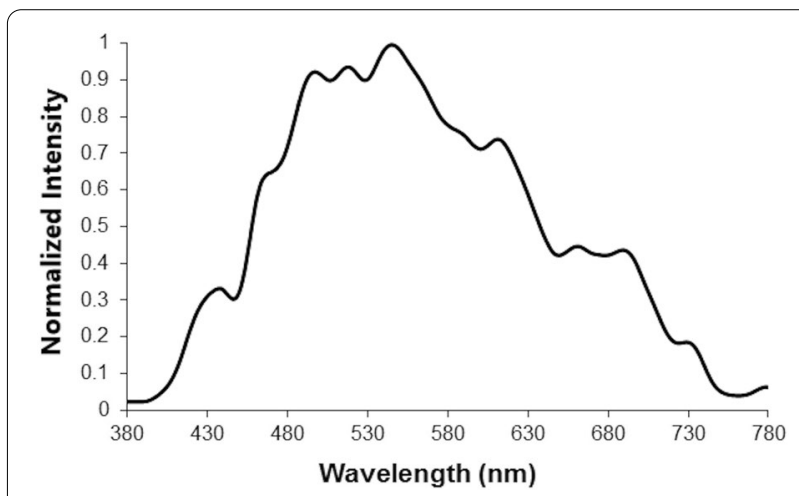

Fig. 1 Spectral power distribution (SPD) of the solid-state plasma light source (LIFI) used in microfading testing experiments. 
non-contact measurement and detection. The illumination fiber is connected to a probe containing three achromatic lenses with focal lengths of 75,75 and $50 \mathrm{~mm}$, which focus the beam to a spot of about $0.5 \mathrm{~mm}$. The collection fiber is connected to a probe holding two achromatic lenses with 50 and $75 \mathrm{~mm}$ focal lengths collecting the scattered light reflected from the sample at an angle of $45^{\circ}$. The estimated illuminance measured at the spot is in the 4.0-6.0 Mlx range. The MFT uses an Ocean Optics (Florida, US) Jaz UV-Vis spectrometer for registering spectral data. The Jaz spectrometer is responsive in the $200-1100 \mathrm{~nm}$ range and has an optical resolution of $\sim 1.5 \mathrm{~nm}$ (FWHM). In addition, a PM100USB power and energy meter from Thorlabs was used to monitor the stability of the generated beam every day before making the measurements. The signal drift recorded in $\mathrm{mW}$, remained within $5 \%$ after 15 min of continuous exposure from any of the two light sources used. After stabilization of the light source, the spectrometer was calibrated using a USRS-99-010 white Spectralon ${ }^{\circledR}$ calibration standard (Labsphere, USA). Visible reflectance spectra were collected using a $100 \mathrm{~ms}$ integration time, 10 average scans and a boxcar width of 20 .

Some examples of the MFT during measurements are presented in Fig. 2 to show the interaction between the illuminated spot and the surface of various objects.

\section{Stereomicroscopy}

A Zeiss SteREO Discovery V12 stereomicroscope (Oberkochen, DE), equipped with a Zeiss AxioCam ERc


Fig. 2 Examples of MFT measurements: a technical drawing from the collection of the Coal Mining Museum in Zabrze, Poland; b large-format pastel artwork on paper by Stanisław Wyspiański (measurement carried out through glass on a vertical surface); $\mathbf{c}$ fragment of the painting "The Ecstasy of St Francis" by El Greco from the Diocesan Museum in Siedlce, Poland; $\mathbf{d}$ area of fresco paintings at the The Church of the Holy Trinity in Lublin, Poland (measurement on a vertical surface) 
5s camera, operating in reflection mode was used to observe and acquire images of the materials used for the direct measurement methods, which included the pinhole in aluminum foil and the materials used as sharp edges. Samples were illuminated using an external LED source with a color temperature of $6200 \mathrm{~K}$.

\section{Characterization of MFT illumination spot}

Several methods were used to characterize the MFT illumination spot with the aim of comparing the results obtained through each technique and discussing their advantages and limitations. The methods were divided into two categories, namely direct and optical imaging. In Table 1 are summarized the considered methods specifying beam diameter, reliability in terms of measurement accuracy (or others parameters) and main features.

\section{Digital photography}

As a preliminary step, images of the MFT illumination beam projected on a piece of white paper were acquired with a Canon $^{\circledR}$ EOS 40D camera (Tokyo, JP) using a Canon Macro Lens EF $100 \mathrm{~mm}$.

\section{Aperture method}

For the aperture method, a homemade pinhole approach was used to determine the MFT illumination beam output intensity distribution. This method uses a relatively smaller size pinhole relative to the expected diameter of the investigated beam. The pinhole is scanned across the beam with the aim of accurately locating its center [26]. The intensity of the beam is measured over the aperture of the pinhole and a plot of intensity as function of beam radius is obtained by scanning the pinhole through the beam. For this purpose, a pinhole setup was prepared using laboratory aluminum foil with thickness $0.030 \mathrm{~mm}$ (Witko, PL). A $2 \times 2 \mathrm{~cm}$ piece of aluminum foil was pierced with the tip of a 25-gauge syringe needle. The aluminum foil pinhole was attached to a MVS005
(Thorlabs, US) vertical stage and a PT1 (Thorlabs, US) horizontal stage to allow movement using a $0.02 \mathrm{~mm}$ step in $\mathrm{x}$ - and $\mathrm{y}$ - directions. The pinhole was placed between the illumination and collection probes. The spectrometer used for these measurements was the USB4000 (Ocean Optics, US). The integration time was $1 \mathrm{~s}$ with 10 average scans and a boxcar width of 20 . The SpectraSuite (Ocean Optics, US) software was used for reflectance spectra acquisition. A schematic diagram of the setup is shown in Fig. 3.

\section{Sharp edge method}

Another direct technique employed in this study was the sharp edge method. This method has been widely used for determining the beam quality in laser applications using a knife-edge approach [26, 33]. It is characterized by its simplicity and its extensive use over a wide range of wavelengths. In this method a sharp edge is translated perpendicular to the direction of propagation of the beam, while a comparison as line scan power differences dependent on the position of the sharp edge provide an accurate measurement of the beam diameter. When the beam is not covered by the material, the measured power reaches its maximum value. In contrast, the power gradually decreases as the material reduces the amount of light reaching the detector by blocking the beam. The first step was to prepare a plot of the recorded signal as a function of the distance travelled by the edge of a specific material. Next, the first derivative of this plot was calculated, which in turn allowed to determine the diameter of the illumination spot. The step used was $0.01 \mathrm{~mm}$. The measurement setup was similar to the one used for the pinhole measurement, the only difference was that instead of a pinhole, the edge of the material was moved along $\mathrm{x}$ - and $\mathrm{y}$ - directions using the translation stages to reduce the amount of light reaching the detector (Fig. 3).

Edges obtained using a safety knife blade, a silicon wafer, muscovite, and Teflon tape were evaluated within

Table 1 Summary of methods used to characterize the illumination spot

\begin{tabular}{|c|c|c|c|c|}
\hline Method & Category & $\begin{array}{l}\text { Estimated beam } \\
\text { diameter }(\mu \mathrm{m})\end{array}$ & Reliability & Features of the method \\
\hline Photography & Imaging & 1200 & Low & Easy to use, inexpensive, simple apparatus - camera or smartphone \\
\hline $\begin{array}{l}\text { Aperture } \\
\text { Aluminium foil }\end{array}$ & Direct & - & Moderate & $\begin{array}{l}\text { Inexpensive, low accuracy with homemade pinhole } \\
\text { Easy to use, inexpensive, material with a well-defined edge, soft- }\end{array}$ \\
\hline $\begin{array}{l}\text { Sharp edge } \\
\text { Razor blade } \\
\text { Silicon wafer } \\
\text { Muscovite } \\
\text { Teflon tape }\end{array}$ & & $\begin{array}{l}590.2 \\
605.9 \\
757.1 \\
610.0\end{array}$ & & ware for data analysis and calculation \\
\hline CMOS camera & Imaging & 702.2 & Moderate & Easy to use, moderate cost, dedicated CMOS detector \\
\hline Laser beam profiler & Imaging & 386.1 & High & Specialist knowledge, costly, advanced equipment - beam profiler \\
\hline
\end{tabular}




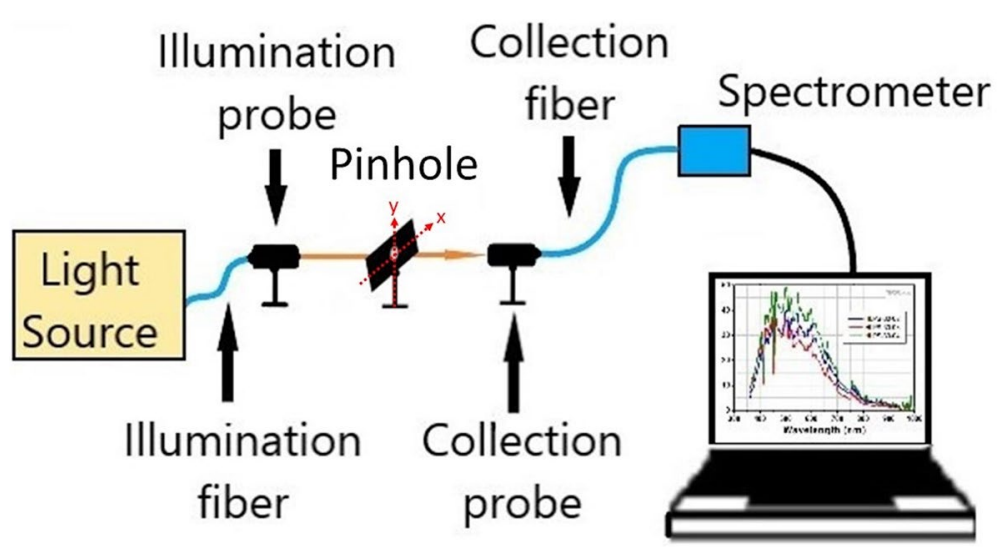

Fig. 3 Experimental setup used to determine the diameter of the MFT illumination beam

this method. The selection of muscovite, silicon and Teflon was based on previous publications in which these materials have been employed in microscopy [34-36], spectroscopy [37, 38], and optics [39, 40] applications. The safety knife blades and the Teflon tape were purchased from a local market in Krakow, Poland. The silicon wafer was produced and made available for research by the Jerzy Haber Institute of Catalysis and Surface Chemistry, Polish Academy of Sciences (Krakow, PL). Muscovite Mica 53000 was purchased from Kremer Pigmente (Aichstetten, DE).

\section{Direct methods models}

The mathematical model followed to determine the diameter of the irradiation beam is the one proposed by Chapple [41], originally used to calculate the irradiance of an ideal laser beam that displays a Gaussian profile. According to Chapple, the irradiance $I(x, y)$ of a laser beam can be described by the following equation:

$$
I(x, y)=I_{0} \exp \left[-\frac{2\left(\left(x-x_{0}\right)^{2}+\left(y-y_{0}\right)^{2}\right)}{r^{2}}\right]
$$

where $I_{0}$ is the peak irradiance at the center of the beam, $x$ and $y$ are the transverse Cartesian coordinates of any point with respect to the center of the beam located at $\left(x_{0}, y_{0}\right)$, and $r$ is the $1 / \mathrm{e}^{2}$ beam radius. The irradiance is replaced by the total power $P_{T}$, since power and irradiance are related by the area, which is a constant factor. Direct methods will result in gradual increases or decreases in total power depending on the position of the attenuation material during a series of step measurements. The derivative of the edge or pinhole data is found using the equation below to obtain a two-dimensional Gaussian profile. The derivative at any data point was performed averaging two adjacent data points derivatives computed using the finite difference method:

$$
\frac{d P_{T}}{d x}=\frac{1}{2}\left(\frac{y_{i+1}-y_{i}}{x_{i+1}-x_{i}}+\frac{y_{i}-y_{i-1}}{x_{i}-x_{i-1}}\right)
$$

the $1 / \mathrm{e}^{2}$ radius was then obtained by fitting a Gaussian function to the data using OriginPro 2021 (OriginLab, USA). The model is equally applicable to a beam of light focused to a small spot.

\section{CMOS camera}

A compact DCC1645C-USB 2.0 (Thorlabs, US) complementary metal-oxide-semiconductor (CMOS) camera was used to record images of the illumination beam and determine its diameter. This camera has a resolution of $1280 \times 1024$ pixels and an exact sensitive area of 4.61 $\mathrm{mm} \times 3.69 \mathrm{~mm}$. This model offers a pixel size of $3.6 \mu \mathrm{m}$ (square) and has a micro lens with a $25^{\circ}$ chief ray angle (CRA) correction. The camera was installed directly in front of the illumination beam at the same position where the pinhole or sharp edge materials were originally placed (Fig. 3). The ThorCam software (Thorlabs, US) was used to provide system control and image acquisition. ImageJ software (National Institutes of Health, Bethesda, MD) was used for image analysis and performing measurements of the size and shape of the spot as well as the intensity of the spot.

\section{CCD camera beam profiler}

A BC106-Vis CCD Camera Beam Profiler (Thorlabs, US) was also used to characterize the illumination spot of the MFT. This camera operates in the $350-1100 \mathrm{~nm}$ wavelength range and has a resolution of $1360 \times 1024$ pixel. The aperture size is 8.77 and $6.60 \mathrm{~mm}$ for width and height, respectively. The pixel size was equal to $6.45 \mu \mathrm{m}$ for both width and height. The filter wheel was rotated to 
set the ND filter with the greatest intensity reduction (40 $\mathrm{dB}$ corresponding to 0.0001 transmittance) in front of the camera aperture to prevent damage to the camera sensor. The signal intensity for the measurements was $100 \mu \mathrm{W}$ and the exposure time was $180 \mathrm{~ms}$.

\section{Results and discussion}

The characterization of the illuminated spot is usually the first step taken by microfading researchers in order to determine the diameter, intensity, and shape of the illumination spot. Typically, once the spot size and shape are characterized, a micro-integrating sphere sensor together with a radiometer are used to determine the intensity of the illuminated spot; this procedure has been described elsewhere $[1,4]$.

In the present work, the first approach used to evaluate the shape and size of the beam was to project the illumination spot of the custom built MFT on a piece of white paper as shown in Fig. 4a. The working distance of about $1.0 \mathrm{~cm}$ typically used during a fading test was also used for these measurements. The diameter of the beam was estimated at $1.2 \mathrm{~mm}$. The configuration of microfading tester used for acquiring the images of the spot is shown in Fig. 3b, c. shows a similar measurement carried out on the analysis spot of a commercially available Oriel 80190 Fading Test System produced by Newport (California, US). In this case, the light exiting the illumination probe was focused to a pinhole size by adjusting the working distance from the edge of the illumination probe to the surface of a semi-transparent glassine paper to $1.0 \mathrm{~cm}$. An image of the spot was then acquired using a Leica MZ-16 microscope equipped with a digital high resolution color camera. The estimated diameter of the illuminated spot was $400 \mu \mathrm{m}$. Visual inspection of the image reveals that the center of the spot receives higher illuminance indicated by color variations that range from bright white passing through yellow, orange and finally red, as one moves from the inner part of the spot out towards its edges.

Some interesting observations can be made after comparing the illumination spot obtained for the custombuilt MFT instrument with the one observed for the commercial version. The latter was developed by Whitmore [1] and was commercially available as the Oriel 80190 fading test system. In general, the use of a digital camera may result in overestimation of the diameter of the illumination beam relative to the image obtained using a microscope. After analyzing and measuring the spot, it was observed that even at the proper working distance a relatively higher size of the spot was obtained relative to the other methods employed. The diameter of the MFT beam acting on the analyzed surface has been reported to be up to $0.5 \mathrm{~mm}[1,3,12,42]$. The $1.2 \mathrm{~mm}$ value obtained using digital photography seemed too large indicating that a different measurement method was necessary. Thus, micrographs can be used to determine the size of the illumination spot since they offer a better alternative to digital photography in terms of accuracy. Following this approach, the pinhole used for determining the size of the illumination beam of the MFT instrument was examined with stereomicrophotography. An example of a microscopic image of the pinhole used is shown in Fig. 5a. Measurements along the $x$ and $y$ axes of the pinhole were 69.85 and $69.50 \mu \mathrm{m}$, respectively. Very accurate measurements are possible when using a combined approach of microscopy and image analysis. In the example presented in Fig. 5a nearly symmetric circle was obtained after comparing measurements of $x$ - and $y$ axes, which have a standard deviation of $0.18 \mu \mathrm{m}$.

Plots of the intensity of the beam measured over the aperture of the pinhole along $\mathrm{x}$ - and $\mathrm{y}$ - axes are presented in Fig. 5. The red and black profiles obtained correspond to unfiltered and filtered beam signals. The filter used was a NE05B $25 \mathrm{~mm}$ absorptive ND filter (Thorlabs, US) a with an optical density of 0.5 . The filter was used to attenuate the beam in order to obtain a closer profile to a Gaussian curve. One of the advantages of this technique is that the position of the pinhole does not need to be known beforehand as it can be located during the measurements [43]. Although a fairly symmetrical hole was pierced on the aluminum foil (Fig. 5a), the plots showed significant deviation from a Gaussian curve. The profiles obtained along the $\mathrm{x}$-axis were similar with and without the use of the filter. However, after inspecting the profiles obtained along the y-axis it can be seen that the ND filter resulted in a different profile showing a decrease in intensity between 200 and $300 \mu \mathrm{m}$. Attenuation of the beam also resulted in a broader beam diameter relative to the measurement performed without a filter. The results obtained here show that the determination can be inaccurate using this direct method. This was likely due to lack of cleanliness when piercing the aluminum foil resulting in microscopic irregularities such as barbs and other material residues inside the pinhole. In addition, manually piercing the aluminum foil with a syringe needle did not provide an accurate optical aperture. The aluminum foil and the detection system used were not optimal for observing subtle intensity differences near the edges of the pinhole. In this configuration, the image of the spot was not uniform, while attempting to fit a Gaussian function to such an irregular curve resulted in unreliable results. Although it seemed like a straightforward approach, a homemade pinhole using aluminum foil was not an adequate method to determine the shape and size of the spot. 

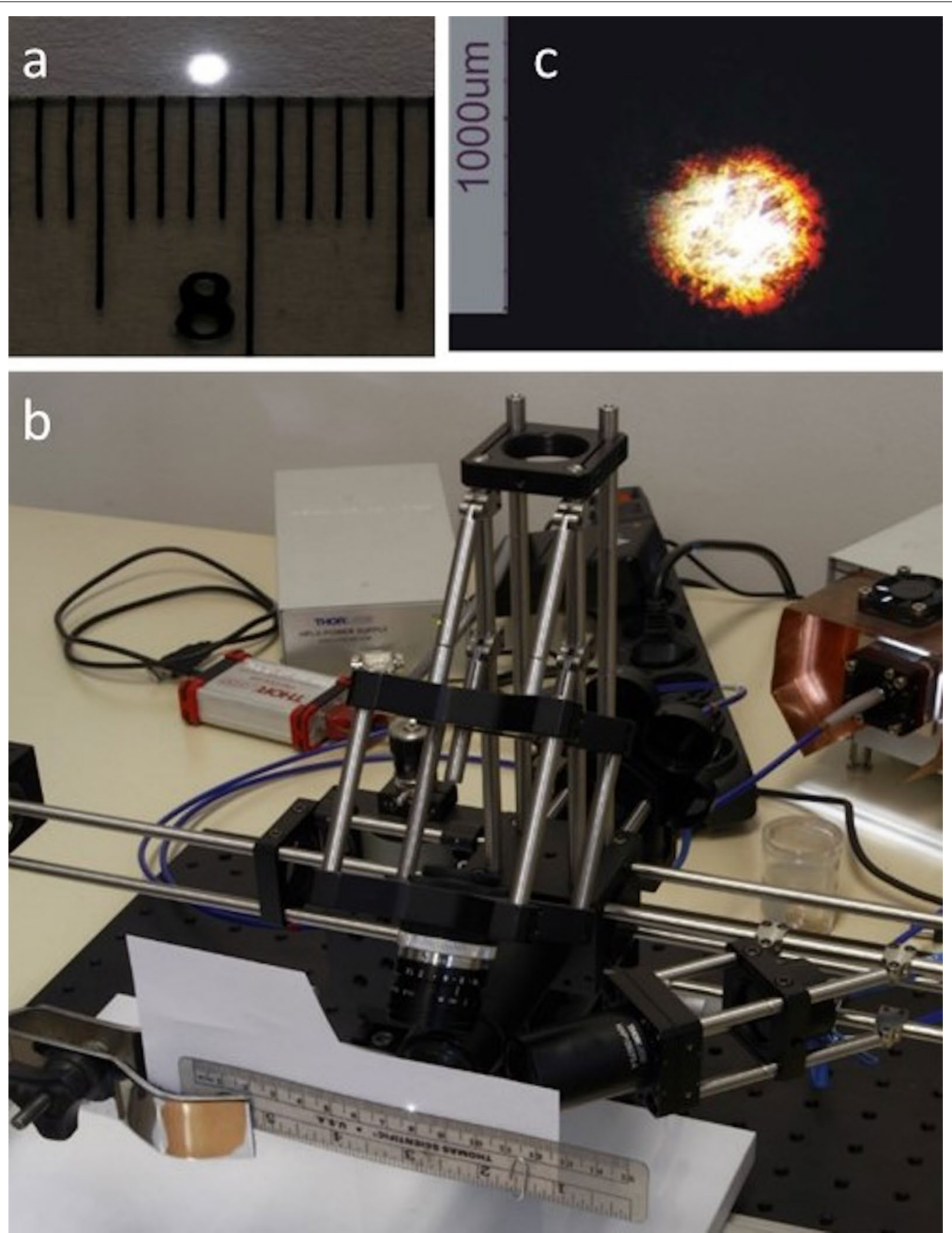

Fig. 4 Digital images of: a illuminated spot of the MFT evaluated in this study; $\mathbf{b}$ setup used to acquire the images of the spot and $\mathbf{c}$ the spot delivered by an Oriel 80190 Fading Test System [42]

While a near top-hat beam shape was observed for the unfiltered measurement along the y- axis, the use of a commercially available pinhole with a consistent diameter and a more stable material is recommended. This will provide a more accurate measurement of the diameter of the beam due to the cleanliness of the material and accuracy of the pinhole.

The sharp edge method was a more reliable alternative to determine the diameter of the illumination spot. A series of measurements of power as a function of position 

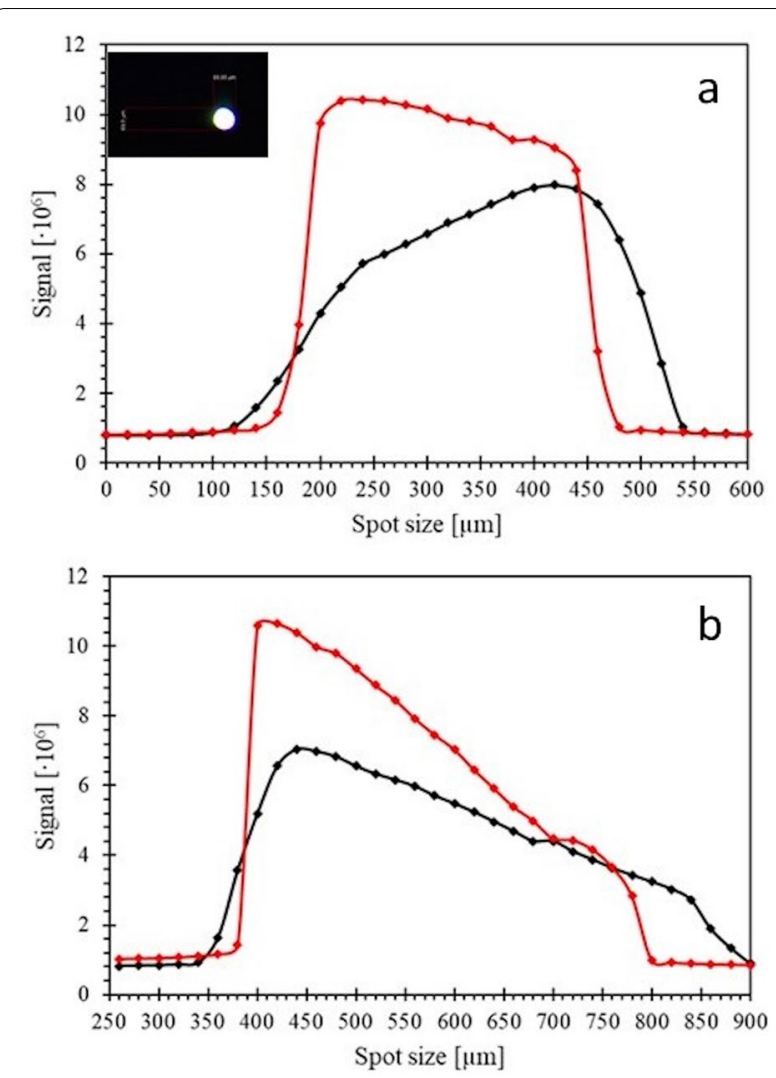

Fig. 5 Beam profile using the aperture method with aluminium foil: a measurement carried out along the $y$ - axis and $\mathbf{b}$ determination performed along the $x$-axis. Red and black lines correspond to unfiltered and filtered beams, respectively

of the razor blade were carried out, starting from a position where the laser beam was not blocked at all, so the measured power was equal to the total power. The final position was chosen at a point where the beam was totally blocked out and the measured power was negligible and remained constant. To obtain the full profile of the beam a displacement of the razor blade of about $1.20 \mathrm{~mm}$ along the $\mathrm{x}$ axis was needed. The characteristic S-shaped curve expected is shown in Fig. 6 a.

Figure $6 \mathrm{~b}$ shows the first derivative (black line) of the measured curve reported in Fig. 6a. The red line shows the Gaussian function fitted to the experimental data. After selecting the appropriate mathematical function, it was possible to determine the parameters necessary for the broad interpretation of the measurements carried out on the illumination spot. These parameters are full-width at half-maximum (FWHM), diameter at the baseline (b), diameter at a power of $13.5 \%(z)$, and diameter for the height of $86.5 \%(w)$. The calculated parameters for the different materials used are summarized in Table 2.

Measurements and calculations performed along $x$ and $y$ - directions provided a $2 D$ representation of the
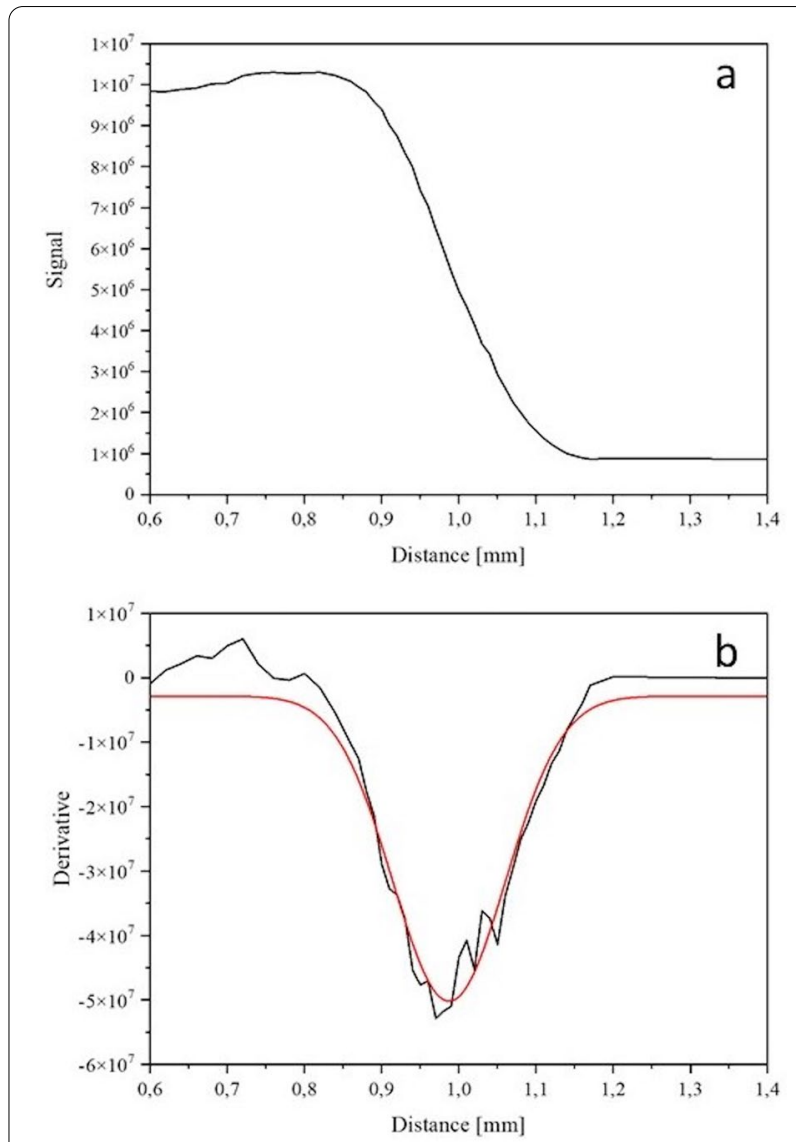

Fig. 6 Example of measurements taken using the sharp edge method razor blade along the $x$ axis: a plot of optical power versus position of the blade and $\mathbf{b}$ calculated diameter of the spot (black line) along with fitted Gaussian function (red line)

MFT illumination spot. The $b$ parameter was considered the most representative value and therefore, it was used as the approximate size of the spot. Average $b$ values of $590.2,605.9,757.1$, and $610.0 \mu \mathrm{m}$ were obtained by using a razor blade, Teflon tape, silicon wafer, and muscovite,

Table 2 Beam diameters in $\mu$ m obtained using various materials with the sharp-edge method

\begin{tabular}{lllrll}
\hline Material & $\begin{array}{l}\text { Direction of } \\
\text { step }\end{array}$ & $\boldsymbol{F W H M}$ & \multicolumn{1}{l}{$\boldsymbol{w}$} & \multicolumn{1}{l}{$\boldsymbol{b}$} & $\boldsymbol{z}$ \\
\hline Razor blade & $\mathrm{x}$ & 172.3 & 97.1 & 537.1 & 292.9 \\
& $\mathrm{y}$ & 223.9 & 126.2 & 643.2 & 382.8 \\
Teflon tape & $\mathrm{x}$ & 214.3 & 121.8 & 606.6 & 364.4 \\
& $\mathrm{y}$ & 214.8 & 136.3 & 605.1 & 360.5 \\
Silicon wafer & $\mathrm{x}$ & 153.6 & 86.6 & 459.5 & 261.3 \\
& $\mathrm{y}$ & 209.3 & 117.9 & 1054.7 & 348.2 \\
Muscovite & $\mathrm{x}$ & 205.1 & 115.6 & 607.4 & 350.4 \\
& $y$ & 228.5 & 128.8 & 612.6 & 281.9 \\
\hline
\end{tabular}


respectively. Some scatter in the data is observed depending on the method used. The estimated average size of the spot was $640.8 \pm 78.0 \mu \mathrm{m}$. An initial inspection of the data suggest that the spot has an elliptical shape, especially for the results obtained with the silicon wafer. In contrast, the differences obtained with the Teflon tape and muscovite are almost negligible indicating that the spot is circular. The calculations made from measurements conducted using a silicon wafer showed higher dispersion relative to the three remaining materials. Although this material showed a very sharp edge when observed under the microscope, the results indicate that it has poor consistency.

In the next stage, the CMOS camera was used to characterize both the illumination and measuring spot. The sensor was placed on the path of the MFT illumination beam and with suitable attenuation images of the spot were recorded. Since the sensor resolution and the size of a single pixel were known, it was possible to determine the size of the spot on the basis of the image recorded. The estimated spot diameter was $702.2 \pm 3.6 \mu \mathrm{m}$ (Fig. 7a). Profiles of the illumination spot in 2D and 3D obtained are presented in Fig. 7b, c, respectively.

Image analysis shows that the MFT illumination beam has a top-hat shape, which indicates that the irradiated area receives a uniform amount of energy throughout the entire area analyzed. A MFT beam previously characterized by Liang et al. also exhibited a top-hat profile along the minor axis and near top-hat shape along the major axis [12]. An evaluation of the MFT optical setup revealed a dependence of the measured signal on the working distance. For this reason, the FWHM was also determined for the collection spot as well as for the common area (illumination and collection) along the two main axes to determine the width and length of the two spots

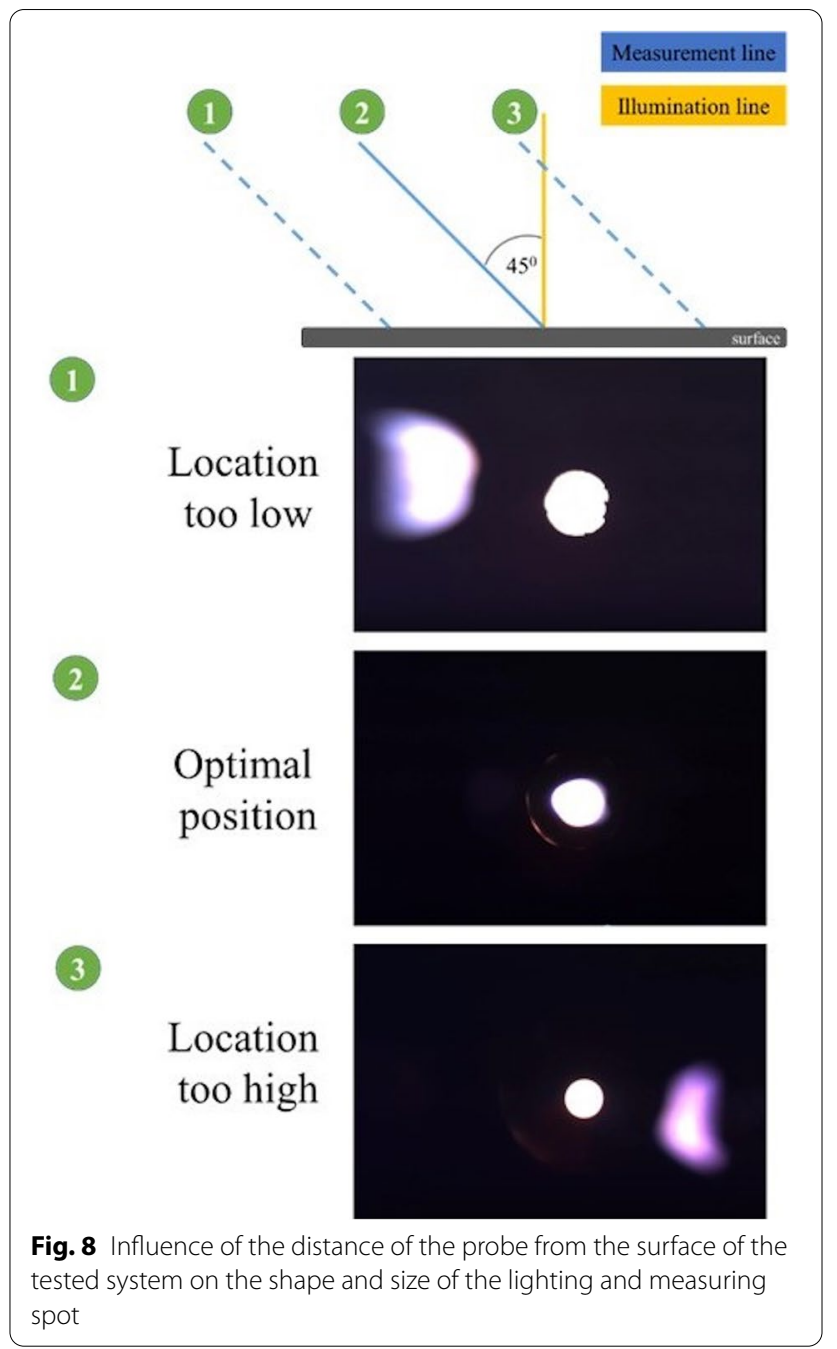

A second light source was used to pass light through the collection fiber in order to determine the size and shape of the collection spot. Figure 8 shows the location of individual spots depending on the distance of
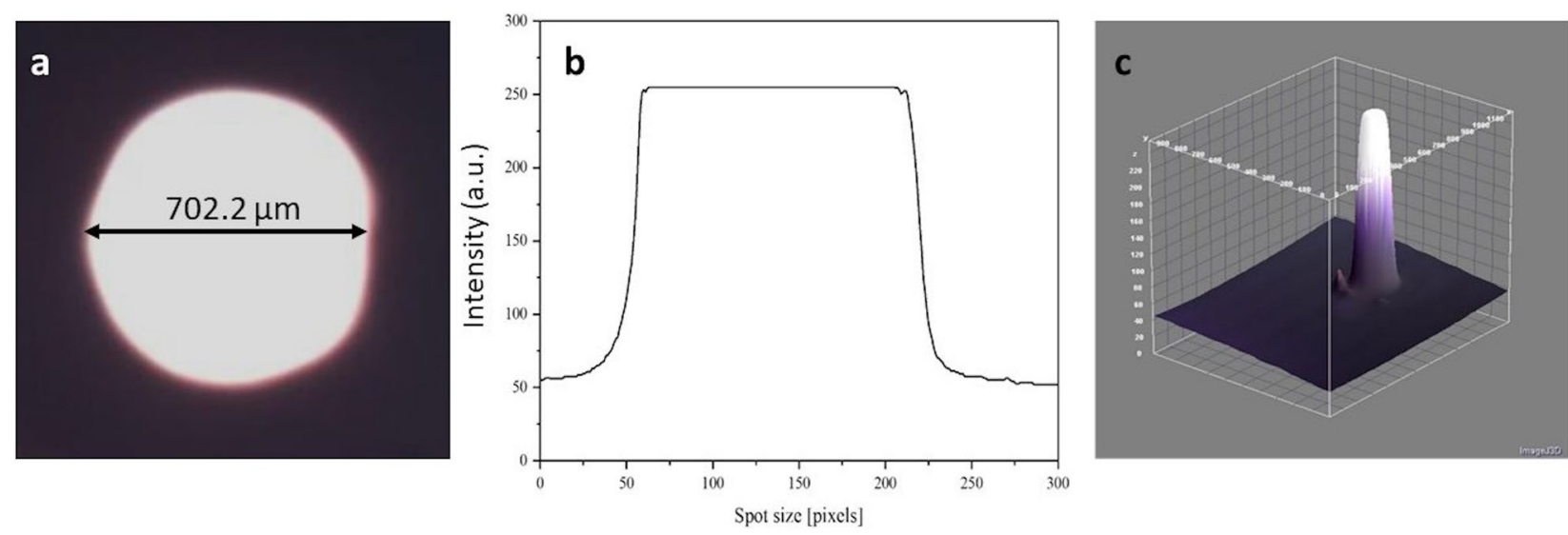

Fig. 7 MFT illumination beam: a CMOS detector image; $\mathbf{b} 2 \mathrm{D}$ profile of the spot; $\mathbf{c} 3 \mathrm{D}$ profile 
the probe from the surface of the tested object. Three MFT optical setup positions are shown, namely lower than optimal, optimal, and higher than optimal. It can be observed that the shape of the collection spot becomes distorted as one gets too close or too far from the optimal position. As one approximates the optimal position, the collection spot starts taking an elliptical shape up to the point of optimal alignment. As expected for this measurement configuration, the optimal optics alignment position is reached when illumination and collection lines meet at 0 and $45^{\circ}$, respectively, relative to the analyzed surface. At this working distance, the illumination spot remains within the collection spot

Further analysis of the illumination and collection areas was conducted using the laser beam profiler. This instrument is typically used in optics and physics laboratories to characterize laser beams. Examples of measurements for illumination and collection spots using this technique are presented in Fig. 9. Figure 9a, b show the images obtained for the illumination spot in 2D and 3D representations, respectively. The illumination beam exhibits uniform distribution of energy over the tested area and has a round nearly top-hat shape (yellow plots) as previously shown by the CMOS camera. The red lines correspond to Gaussian fits of the data. The main part of illumination energy is concentrated along the main tophat peaks and weaker wavelets of about $50 \mu \mathrm{m}$ around the main peak can be recognized. The $2 \mathrm{D}$ and $3 \mathrm{D}$ images obtained for the collection spot are shown in Fig. 9c, d, respectively. The oval shape of this spot becomes evident after inspecting the image, which shows presence of a major and a minor axis. The energy profile of the collection spot along with its 3D plot confirm its top-hat beam shape.

An image of the common area of illumination and collection captured with the CMOS camera is shown in Fig. 9e. The images obtained with the CMOS camera and the laser beam profiler were further analyzed using ImageJ to obtain a representation of the common area irradiated and measured using MFT. The shape and size of the illumination spot was a circle with a diameter of $386 \mu \mathrm{m}$, while the diameter along $\mathrm{x}$ - and $\mathrm{y}$ - axes of the collection spot were 445 and $629 \mu \mathrm{m}$, respectively. A schematic representation of the two spots at the optimal working distance is shown in Fig. 9f. A similar analysis of the tested area was conducted by Lerwill et al. [21]. The authors carried out a similar procedure to verify confocality of the optical setup by focusing both beams onto a CCD sensor. In this way, they were able to indicate the sampling area of the collection probe and identify the region where fading takes place. The results from the present study are in agreement with those obtained by Lerwill and co-workers, where the maximum signal detected by the spectrometer is associated with a reproducible spot size and low variation in the calculated fading.

\section{Conclusion}

This study has evaluated the performance of various conventional and imaging methods for the characterization of MFT illumination and measurement spots. The advantages and disadvantages of several methods used to determine the beam shape and intensity profiles have been described. Although a preliminary view of the appearance and size of the illumination beam can be obtained using digital photography, this approach alone cannot be used to determine the actual shape and the size of the spot. A homemade pinhole was tested for the aperture method, but no reliable results are reported mainly due to error associated with manual piercing of the aluminum foil. For this reason, the use of a machine-made commercial pinhole is recommended over a homemade one. In general, sharp-edge methods enable calculation of the size of the illumination and collection spots. For this purpose, a material with a well-defined edge is essential, otherwise higher dispersion of the beam could be observed due to the use of an irregular edge or a material with low beam attenuation power. The results obtained with the four materials used in the current study show good agreement. Therefore, the use of readily available materials, such as a razor blade or Teflon tape, is recommended. An imaging approach using a CMOS camera or a laser beam profiler should be used as a complementary method to the direct determination method. The laser beam profiler provided an accurate way of measuring and characterizing the illumination and collection spots of the MFT. However, one disadvantage is its relatively higher cost when compared to other techniques used in this study. An adequate characterization of illumination and collection spots is important since during a MFT measurement the surface of the analyzed object has an evident influence on the measurement and an object containing extremely sensitive materials may experience a microscopic but noticeable change as a result of testing. The accurate characterization of both beams allows for a better understanding of the tested system and enhanced knowledge about the interaction between light and the surface under investigation. From our experience, it would be advisable to first acquire images of the MFT illumination beam with a CMOS camera followed by a determination of the beam diameter using a direct method, more specifically one involving a sharp-edge technique. Although characterization of the illumination spot is a time-consuming task, the authors recommend conducting and documenting these measurements subject to frequency of use of the instrument and any significant hardware changes. 

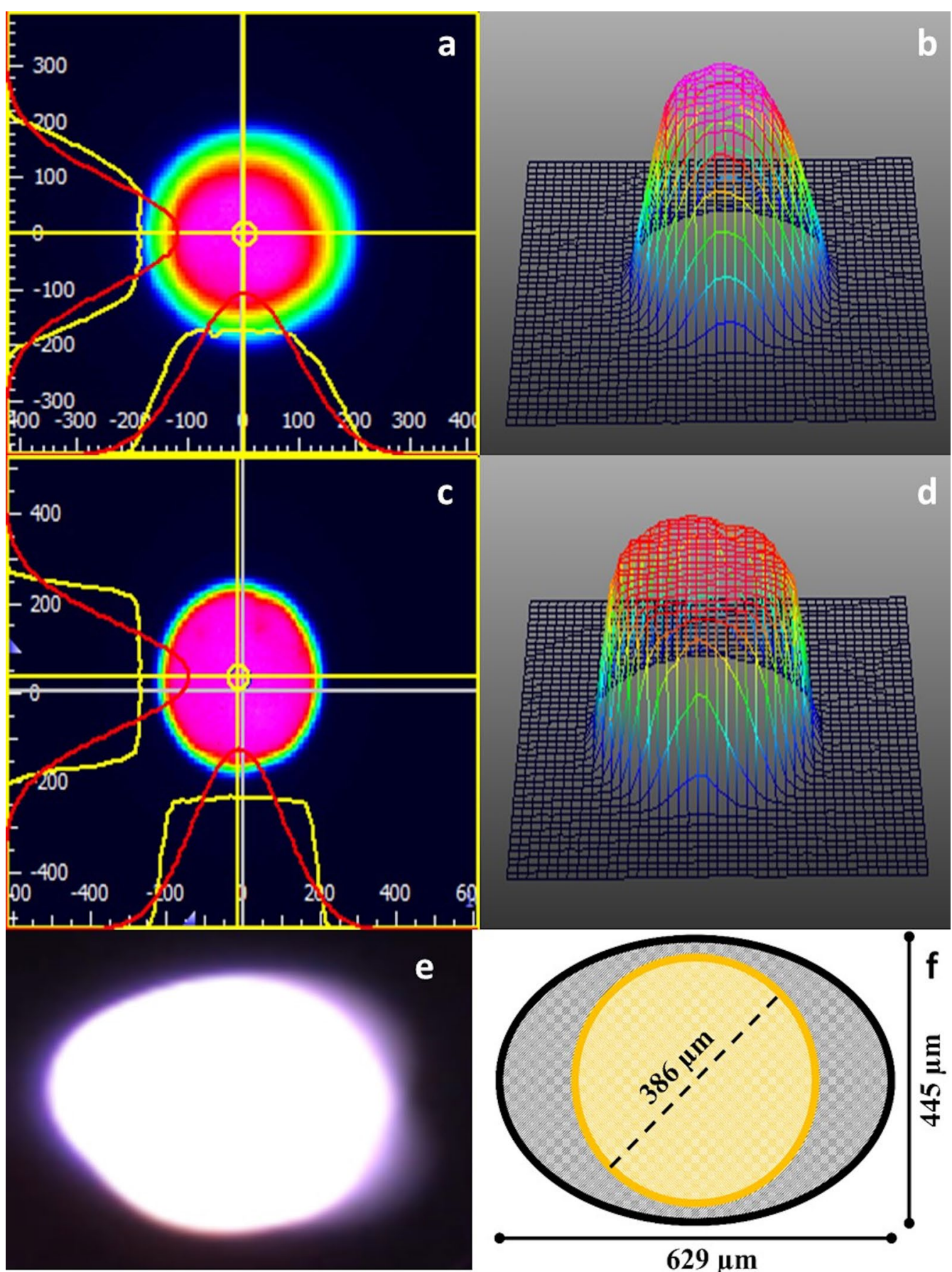

Fig. 9. Laser beam profiler measurements: a $2 D$ image of the illumination spot showing energies along $x$ - and $y$-axes; $\mathbf{b} 3 D$ view of the illumination spot; $\mathbf{c} 2 \mathrm{D}$ image of the collection spot showing energies along $\mathrm{x}$ - and $\mathrm{y}$-axes; $\mathbf{d}$ 3D view of the collection spot; $\mathbf{e}$ image of the common area of illumination and collection captured with the CMOS camera; $\mathbf{f}$ estimated sizes and shapes of illumination spot (yellow) and collection spot (grey) 


\section{Abbreviations}

CCD: Charge coupled device; CMOS: Complementary metal oxide semiconductor; FWHM: Full width at half maximum; MFT: Microfading testing; LED: Light emitting diode.

\section{Acknowledgements}

The authors are very grateful to Professor Krzysztof Dzierżęga from the Faculty of Physics, Astronomy and Applied Computer Science of the Jagiellonian University in Krakow for allowing us to borrow the beam profiler used in our study.

\section{Authors' contributions}

PŚ: conceptualization, methodology, validation, investigation, writing original draft. MG: methodology, validation, writing original draft. JdH: conceptualization, methodology, project leader, writing-original draft. All authors read and approved the final manuscript.

\section{Funding}

This publication is financed by the Research Excellence Initiative of the University of Silesia in Katowice, Poland.

\section{Availability of data and materials}

The datasets used and/or analyzed during the current study are available from the corresponding author on reasonable request.

\section{Declarations}

\section{Competing interests}

The authors declare no conflict of interest.

\section{Author details}

${ }^{1}$ Institute of Chemistry, Faculty of Science and Technology, University of Silesia, 40006 Katowice, Poland. ${ }^{2}$ Department of Physics, University of Milan, Via Celoria, 16, 20133 Milan, Italy. ${ }^{3}$ Laboratory of Analysis and Non-Destructive Investigation of Heritage Objects, National Museum in Krakow, 31109 Krakow, Poland.

\section{Received: 26 April 2021 Accepted: 25 June 2021}

Published online: 03 July 2021

\section{References}

1. Whitmore PM, Pan X, Bailie C. Predicting the fading of objects: identification of fugitive colorants through direct nondestructive lightfastness measurements. J Am Inst Conserv. 1999;38:395-409.

2. Connors SA, Whitmore PM, Roger S, Keyes RS, Coombs E. The identification and light sensitivity of Japanese woodblock print colorants the impact on art history and preservation. In: Jett P, Winter J, McCarthy B, editors. Scientific Research on the Pictorial Arts of Asia, Proceedings of the Second Forbes Symposium at the Freer Gallery of Art. Washington: Archetype; 2005. p. 35-47.

3. Ford B. Non-destructive microfade testing at the National Museum of Australia. AICCM Bull. 2009;32:54-64.

4. del Hoyo-Meléndez JM, Mecklenburg MF. A survey on the lightfastness properties of organic-based Alaska Native artifacts. J Cult Herit. 2010;11:493-9.

5. Columbia MR, Smith GD, Hoevel C, Messier P. The application of microfadeometric testing to mounted photographs at the Indianapolis Museum of Art. Microsc Microanal. 2013;19(S2):1412-3.

6. Ford B, Druzik J. Microfading: the state of the art for natural history collections. Coll Forum. 2013;27(1):54-7.

7. del Hoyo-Meléndez JM, Gondko K, Mendys A, Król M, Klisińska-Kopacz A, Sobczyk J, Jaworucka-Drath A. A multi-technique approach for detecting and evaluating material inconsistencies in historical banknotes. Forensic Sci Int. 2016;266:329-37.
8. del Hoyo-Meléndez JM, Świt P, Sobczyk J. Measuring photostability through glass: the application of microfading testing to the study of framed pastel artworks. Color Technol. 2018;134(6):411-22.

9. Ford B, Smith N. The development of a significance and risk-based lighting framework at the National Museum of Australia. AICCM Bull. 2009;32:80-6.

10. Tse S. Microfade testing for heritage institutions: a Canadian experience. Stud Conserv. 2019;64(6):337-51.

11. Ford BL. A microfading survey of the lightfastness of blue, black and red ballpoint pen inks in ambient and modified environments. AICCM Bull. 2018;39(1):26-34.

12. Liang H, Lange R, Lucian A, Hyndes P, Townsend, JH, Hackney, S. Development of portable microfading spectrometers for measurement of light sensitivity of materials. In: International Council of Museums, Committee for Conservation (ICOM-CC) Triennial Conference. Lisbon: ICOM-CC; 2011. p. 1-7.

13. Daher C, Tournié A, Sauvagnargues F, Andraud F, Cuisin J, Illes V, Kissel É. Colored feathers in museum collections: a spectroscopic study of 3 bio-pigments and their lightfastness. J Cult Herit. 2020;45:59-70.

14. Beltran VL. Advancing microfading tester practice. Los Angeles: Getty Conservation Institute; 2019.

15. Lauwers D, Hutado AG, Tanevska V, Moens L, Bersani D, Vandenabeele P. Characterisation of a portable Raman spectrometer for in situ analysis of art objects. Spectrochim Acta A. 2014;118:294-301.

16. Mantler M, Klikovits J. Analysis of art objects and other delicate samples: is XRF really non-destructive? Powder Diff. 2004;19:16-9.

17. Targowski $P$, Iwanicka M. Optical coherence tomography: its role in the non-invasive structural examination and conservation of cultural heritage objects-a review. Appl Phys A. 2012;106:265-77.

18. Gaudioso R, Dell'Aglio M, De Pascale O, Senesi GS, De Giacomo A. Laser induced breakdown spectroscopy for elemental analysis in environmental, cultural heritage and space applications: a review of methods and results. Sensors. 2010;10:7434-7368.

19. Lerwill A, Townsend J, Liang H, Hackney S, Thomas J. A versatile microfadometer for lightfastness testing and pigment identification. In: Fotakis C, Pezzati L, Salimbeni R, editors. Proceedings of SPIE. Bellingham: The International Society for optics and photonics; 2007.

20. Saunders D. Photographic flash: threat or nuisance? Natl Gallery Tech Bull. 1995;16:66-72.

21. Lerwill A, Townsend JH, Liang H, Thomas J, Hackney S. A portable micro-fading spectrometer for versatile lightfastness testing. E Preserve Sci. 2008;5:17-28.

22. Cerniglia D, Pantano A, Mineo C. Influence of laser beam profile on the generation of ultrasonic waves. Appl Phys A. 2011;105:959-67.

23. Karsai S, Pfirrmann G, Hammes S, Raulin C. Treatment of resistant tattoos using a new generation Q-switched Nd:YAG laser: influence of beam profile and spot size on clearance success. Lasers Surg Med. 2008;40:139-45.

24. Suzaki Y, Tachibana A. Measurement of the $\mu \mathrm{m}$ sized radius of Gaussian laser beam using the scanning knife-edge. Appl Opt. 1975;14:2809-10.

25. Dickey FM. Laser beam shaping: theory and techniques. Boca Raton: Taylor and Francis; 2000.

26. Marshall WJ. Two methods for measuring laser beam diameter. J Laser Appl. 2010;22(4):132-6.

27. Golnabi H, Haghighatzadeh A. Beam shape and intensity profile of a fiber-bundle prism-coupled waveguide. Opt Lasers Eng. 2010;48(6):720-6.

28. Lednev V, Pershin SM, Bunkin AF. Laser beam profile influence on LIBS analytical capabilities: single vs. multimode beam. J Anal At Spectrom. 2010:25:1745-57

29. Hossain MA, Canning J, Cook K, Jamalipour A. Smartphone laser beam spatial profiler. Opt Lett. 2015:40(22):5156-9.

30. Langer $G$, Hochreiner A, Burgholzer P, Berer T. A webcam in Bayermode as a light beam profiler for the near infra-red. Opt Lasers Eng. 2013;51(5):571-5.

31. Łojewski T, Thomas J, Gołab R, Kawałko J, Łojewska J. Light ageing with simultaneous colorimetry via fibre optics reflection spectrometry. Rev Sci Instrum. 2011;82(7):76102-5.

32. Pesme C, Lerwill A, Beltran V, Druzik J. Development of contact portable microfade tester to assess light sensitivity of collection items. J Am Inst Conserv. 2016:55(2):117-37. 
33. González-Cardel M, Arguijo P, Díaz-Uribe R. Gaussian beam radius measurement with a knife-edge: a polynomial approximation to the inverse error function. Appl Opt. 2013;52(16):3849-55.

34. Gu M, Sheppard CJR. Effects of defocus and primary spherical aberration on images of a straight edge in confocal microscopy. Appl Opt. 1994;33(4):625-30.

35. Haselton HT, Cygan GL, Jenkins DM. Experimental study of muscovite stability in pure $\mathrm{H}_{2} \mathrm{O}$ and 1 molal $\mathrm{KCl}-\mathrm{HCl}$ solutions. Geochim Cosmochim Acta. 1995:59(3):429-42.

36. Zhang J, Li J, Han Y. Superhydrophobic PTFE surfaces by extension. Macromol Rapid Commun. 2004;25:1105-8.

37. Oberg KA, Palleros DR. Teflon tape as a sample support for IR spectroscopy. J Chem Educ. 1995;72(9):857-9.

38. Karabudak E, Kas R, Ogieglo W, Rafieian D, Schlautmann S, Lammertink RGH, Gardeniers HJGE, Mul G. Disposable attenuated total reflectioninfrared crystals from silicon wafer: a versatile approach to surface infrared spectroscopy. Anal Chem. 2013;85:33-8.

39. Wright FE. The splitting of mica (muscovite) for optical purposes. Am J Sci. 1939;237(10):736-41.
40. Lal K, Niranjana S, Goswami N. New and accurate technique for determination of orientation of the straight edges of single crystal wafers. Rev Sci Instrum. 1988:59(8):1409-11.

41. Chapple PB. Beam waist and $\mathrm{M}^{2}$ measurement using a finite slit. Opt Eng. 1994:33(7):2461-6.

42. del Hoyo-Meléndez JM. A study on the action of light on objects of cultural interest by evaluating the light levels in the museum and performing micro-fading tests of materials. Valencia: PhD thesis Universidad Politécnica de Valencia, 2010.

43. Shayler PJ. Laser beam distribution in the focal region. Appl Opt. 1978;17(17):2673-4

\section{Publisher's Note}

Springer Nature remains neutral with regard to jurisdictional claims in published maps and institutional affiliations.

\section{Submit your manuscript to a SpringerOpen ${ }^{\odot}$ journal and benefit from:}

- Convenient online submission

- Rigorous peer review

- Open access: articles freely available online

- High visibility within the field

Retaining the copyright to your article

Submit your next manuscript at $\boldsymbol{\nabla}$ springeropen.com 